\title{
Do allelic variants of SLC6A14 predispose to obesity?
}

\author{
Hemant K. Tiwari ${ }^{1,2}$ and David B. Allison ${ }^{1,3}$ \\ ${ }^{1}$ Department of Biostatistics, Section on Statistical Genetics, \\ ${ }^{2}$ Department of Pathology, and \\ ${ }^{3}$ Clinical Nutrition Research Center, Department of Nutrition Sciences, University of \\ Alabama at Birmingham, Birmingham, Alabama, USA
}

\begin{abstract}
Obesity is arguably the world's most prevalent nutritional disorder and is a substantial contributor to morbidity and early mortality. Obesity is known to have a strong genetic component, but the specific influential genes in humans are largely unknown. A new paper describes a genetic variant that appears as though it may cause some people to be fatter or thinner than others (see the related article beginning on page 1762). This commentary considers the strength of the evidence in support of this finding and discusses additional research questions that should be addressed in further evaluations of this genetic variant as a putative contributor to human obesity.
\end{abstract}

J. Clin. Invest. 112:1633-1636 (2003). doi:10.1172/JCI200320448.

The photograph courtesy of the U.S. National Library of Medicine's historic image archive (Figure 1) is entitled $D r$. E. Brown, the Largest Man in America. From a Portrait by Mr. J.R. Dix. Dr. Brown appears to be a late 19 th-century man. Although a man of his size would have been rare in any day, tragically, he would be far less rare today than in his own time. Rates of obesity in general and severe obesity in particular have continued to climb (1). Many people believe that we will best be able to address the problem of obesity when we better understand the roots of its origins. Beyond the near-tautological insight from the first law of thermodynamics that obesity is the result of taking in more energy than we expend, lit-

Address correspondence to: David B. Allison, Section on Statistical Genetics, Department of Biostatistics, Ryals Public Health Building, Suite 327, University of Alabama at Birmingham, 1665 University Boulevard, Birmingham, Alabama 35294, USA. Phone: (205) 975-9169; Fax: (205) 975-2540; E-mail: Dallison@UAB.edu.

Conflict of interest: The authors have declared that no conflict of interest exists. Nonstandard abbreviations used: quantitative trait locus (QTL); sibling pair (sibpair); solute carrier family 6 member 14 (SLC6A14); single-nucleotide polymorphism (SNP); maximum likelihood score (MLS); waist-to-hip ratio (WHR). tle is unequivocally known about the specific factors that cause variations in adiposity among most humans. We now look to genomic research as one place to seek such answers.

For the last decade or so, a popular strategy that has held high hope for finding genes influencing traits like fatness in humans is the so-called genome scan for linkage. In this method, the genotypes of related individuals are assessed at numerous (hundreds) of roughly evenly spaced points (markers) along the genome. Then, at each point, a statistical test is performed to evaluate whether there is convincing evidence that the degree of phenotypic similarity between any given pair of related individuals is associated with their degree of genotypic similarity at the location in question. If so, we state that there is evidence of linkage. As we write this commentary, a meeting is being held at the National Institutes of Health to examine some issues related to the use of phenotyping in genetic studies of obesity, particularly what traits should be measured and how best to measure them. The rationale for this conference, as put forward by the National Institute of Diabetes and Digestive and Kidney Diseases, is that "the genetics of human obesity has been very intensively studied, with at least ten genome scans already published. These scans have yielded a large number of suggestive genetic linkage findings, with a moderate amount of replication of findings among independent studies. However, these studies have yet to result in the identification of many of the genes predisposing to obesity" (2). This is in contrast to the relative success that has been obtained in identifying certain rare Mendelian forms of obesity such as Bardet-Biedl syndrome. Despite recent successes in implicating specific genes and genomic regions in obesityrelated phenotypes in humans, the genes predisposing individuals to the common non-Mendelian form of obesity remain unknown. Chagnon et al. recently stated "QTLs [quantitative trait loci] reported from animal models currently number 168; there are 68 human QTLs for obesity phenotypes from genome-wide scans. Additionally, significant linkage peaks with candidate genes have been identified in targeted studies. Seven genomic regions harbor QTLs replicated among two to five studies. Attempts to relate DNA sequence variation in specific genes to obesity phenotypes continues to increase, with 222 studies reporting positive associations with 71 candidate genes" (3). However most of these associations have not been convincingly replicated (4-6).

Found: a putative obesity susceptibility gene. It seems likely that the predisposition to obesity involves multiple genes, environmental factors, and developmental processes, and their interactions $(7,8)$. The specific genes and their relative potency probably differ among populations and ethnic groups. In a previous genome scan of Finnish obese nuclear families, using an affected sibling par (sibpair) design, Suviolahti and colleagues identified a locus on chromosome Xq24 with a strong link to obesity (9). In this issue of the JCI, this talented group of investigators has demonstrated, using clever and thorough experimental design, the localization of a putative obesity susceptibility gene, solute carrier family 6 


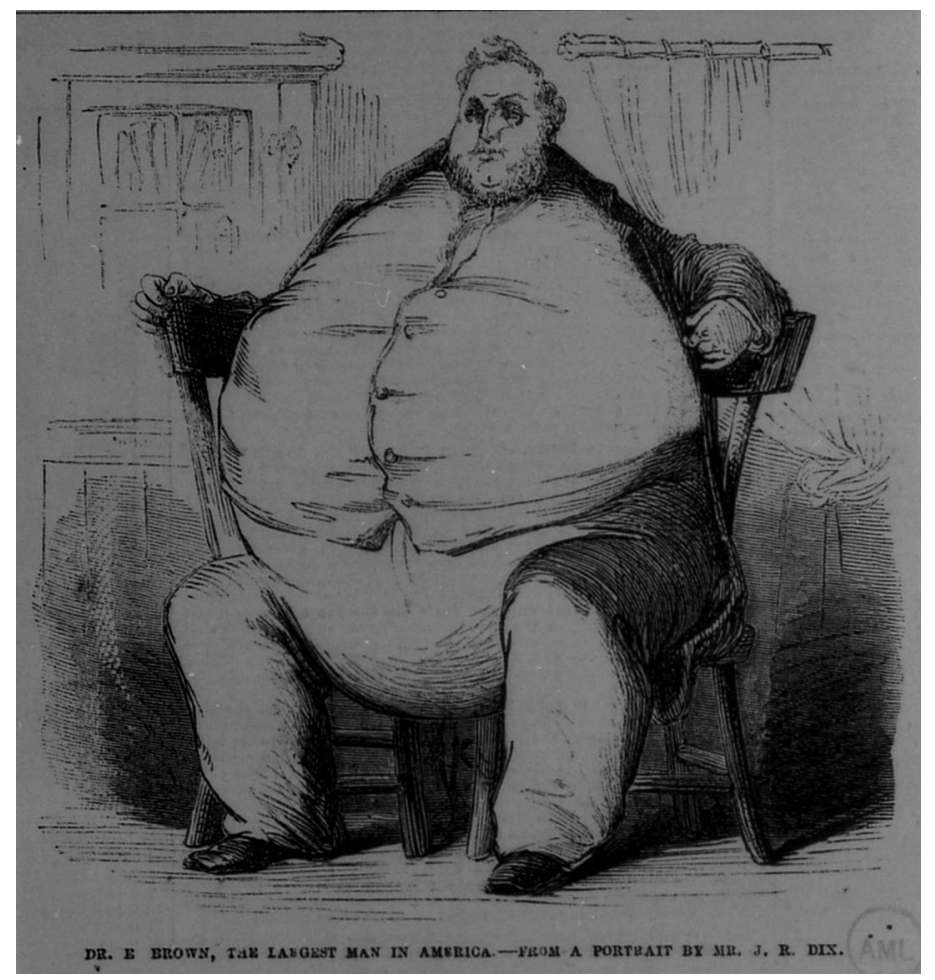

\section{Figure 1}

Dr. E. Brown, the Largest Man in America. - From a Portrait by Mr. J.R. Dix. Image courtesy of the U.S. National Library of Medicine.

member 14 (SLC6A14), on the X chromosome in a Finnish population (10). Fine mapping of the original $15-\mathrm{Mb}$ linkage region on Xq24 for 11 positional and functional candidate genes was achieved using a combination of nine microsatellite markers and 36 single-nucleotide polymorphisms (SNPs) in 218 obese Finnish sibpairs (body mass index $[\mathrm{BMI}]>30 \mathrm{~kg} / \mathrm{m}^{2}$ ). Using haplotype analysis, the authors reduced the linkage region from $15 \mathrm{Mb}$ to $4 \mathrm{Mb}$. In this $4-\mathrm{Mb}$ region, they identified three functional candidate genes, AGTR2, SLC6A14, and SLC25A5, which were tested for association in 117 cases and 182 controls. Significant evidence for association was observed for a SNP in the SLC6A14 gene region and also with the SNP haplotypes of the same gene. The authors further investigated SLC6A14 using 837 cases and 968 controls from Finland and Sweden, and a significant difference in allele frequency between obese and nonobese individuals was observed. They observed reduced evidence of significance in a combined data set $(P=0.0007$ in the Finnish population vs. $P=0.003$ in the combined Finnish and Swedish population), which is not surprising and could be due to genetic heterogeneity. The SLC6A14 gene is a likely candidate gene for obesity, as it is related to serotonin and serotonergic receptor mechanisms that have been implicated in the control of appetite and body weight $(11,12)$.

Confirming the link. The first step in any linkage study is to establish linkage to certain genomic regions using sibpairs or an extended families design. However, it is possible that the initial linkage results represent false positives, and therefore other sources of evidence are required in order to determine which linkage results are most likely to harbor genuine susceptibility loci. This can be accomplished by replicating the linkage results by association analysis to compare the frequency of the putative causal mutation in affected individuals and in appropriate controls (6). The relevance of the detected mutations is confirmed with additional association studies in both the original and independent populations, as well as with functional assays in vitro (expression studies in different cell lines) and in vivo (transgenic and knockout animal models). A procedure designed to identify the causal gene(s) of a particular phenotype within a QTL (13) is summarized in Figure 2.

In their effort to identify a susceptibility gene for adiposity, Suviolahti et al. (10) have used a standard research design (as depicted in Figure 2), and their results are impressive. However, future functional analysis in vitro and in vivo may lead to a better understanding of the causal influence (or lack thereof) of SLC6A14 on the obesity phenotype.

Other related findings. There are very few reports describing significant linkage of BMI with markers on the $\mathrm{X}$ chromosome (10, 14-16). Using sibpair data, Hagar et al. (14) reported linkage of BMI to markers on the $\mathrm{X}$ chromosome with test statistics, denoted as maximum likelihood score (MLS) statistics, as high as 2.42 , which is generally taken to be "suggestive" evidence for linkage (17). Price et al. (15) tested for evidence of linkage of five adiposity-related traits (BMI, percent body fat, hip and waist circumferences, and waist-to-hip ratio (WHR)] to genetic variation in the $\mathrm{X}$-chromosome in two cohorts of EuropeanAmerican and African American pedigrees. Suggestive linkage was found for WHR in women only. The suggestive linkage was $14-34 \mathrm{cM}$ distal from the locus on Xp22 identified by Hagar et al. Stone et al. (16) also found suggestive linkage in the same genomic region, Xp21-22, as did Hagar et al. The genome-wide scan of Finnish sibpairs reported significant linkage between BMI and markers in a region on Xq24 (10). Clearly, the divergent results of Hager et al. and Suviolahti et al. illustrate the problems associated in replicating linkage studies of complex diseases such as obesity.

Significant evidence of association for an SNP3 of SLC6A14 gene $(P=0.0002)$ with BMI was observed using a casecontrol sample of only male subjects from the Finnish population with the G-allele associated with a significantly greater risk of being obese. However, an attempt to replicate the SNP3 association using an independent sample of 
1. Genome scan

2. Fine mapping

\section{Resequencing/ association analyses}

\section{anses \\ 4. Functional assays}

(n)
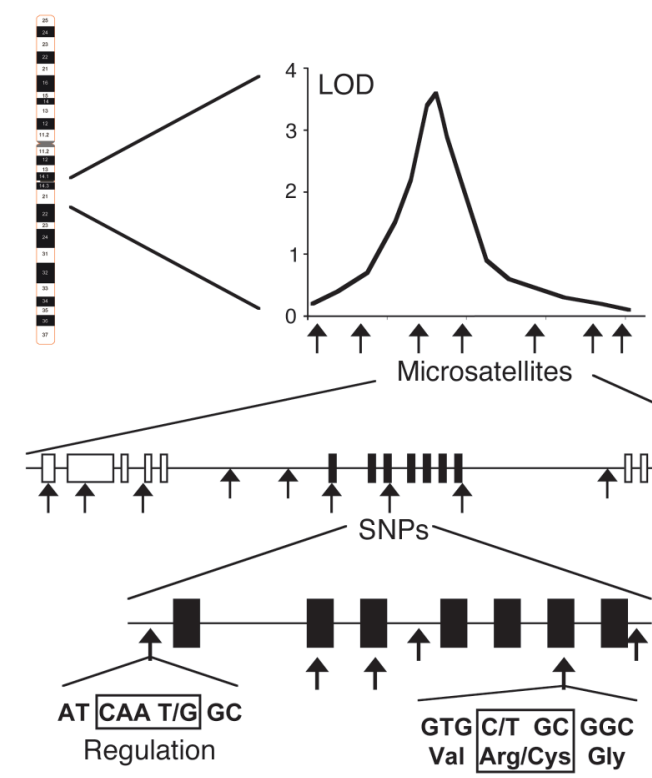

Microsatellites
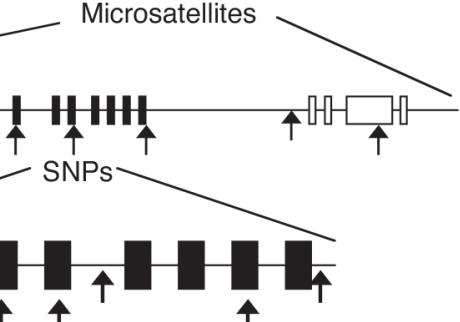

$\uparrow$

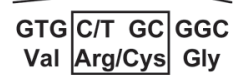

Val Arg/Cys Gly

Structure

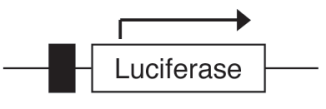

In vitro

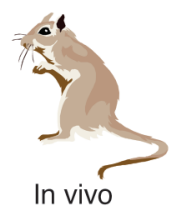

In vivo
Figure 2

Schematic presentation of the positional cloning strategy. The initial genome-wide linkage scan is followed by fine mapping with additional microsatellite and single SNP markers. Genes showing significant associations with the SNPs are resequenced to identify relevant DNA sequence variants in the study population. The relevance of these mutations is verified with additional association studies in other populations (replications) and using in vitro and in vivo functional studies. Modified with permission from Circulation (22). LOD, log (base 10) of the odds ratio. obese and normal controls from combined data from the Swedish and Finnish populations gave contradictory results, namely, the $\mathrm{C}$-allele (rather than G-allele) was found to be associated with a higher risk of being obese rather than non-obese $(P=0.003)$. A similar result was observed when analyzing female subjects only.

There are several reasons that may explain why this replication failed. First, genetic heterogeneity could have been a driving force in changing the direction of the results observed. Second, it is evident from the results that there is a sex-specific component in the predisposition to obesity. To avoid genetic heterogeneity, one should first try to replicate significant findings utilizing a population with the same genetic background. Because males were used in initial association study, it would have been better to replicate the significance of an association by using an independent sample set of males from the Finnish cohort.

The results reported by Suviolahti and colleagues in this issue (10) raise a number of questions. First and foremost, is the polymorphism studied causative of variations in adiposity? Page et al. (18) outline a line of think- ing based on a Popperian falsification framework to help address such questions. In this framework, one successively tries to disprove one's own hypothesis. The repeated inability to disprove the hypothesis is considered as evidence in support of the theory. Clearly, a number of competing explanations need to be ruled out before we can definitively accept the association of the polymorphism under study with BMI as indicative of causation. Such competing explanations include, but are not limited to, the possibility that the polymorphism studied is in tight linkage disequilibrium with a causative polymorphic locus, stochastic variation, and spurious association due to disequilibrium between the polymorphism studied and unlinked causative loci. This final possibility is often attributed to concerns about admixture and thought to be of minimal concern in reputedly more homogeneous populations such as the Finns (19). However, assortative mating - a form of nonrandom mating in which the probability of two individuals mating with each other depends on functions of their joint phenotypic characteristics - can also produce such spurious associations. Positive assortment - where- by people mate with individuals that are phenotypically more similar to themselves (i.e., "birds of a feather flock together") - is believed to be the most common form of mating. The Finns (like most populations) have clearly been shown to assortatively mate for obesity-related phenotypes (20).

Second, if the association is causal, what is the extent of the effect? Because of both the phenotypically selected nature of the sample and the bias inherent in estimating the magnitude of effects when those effects have been found as a result of conducting a genome scan (21), the magnitude of the putative effect of the SLC6A14 locus is difficult to estimate from the present study (10) and might be best addressed in an independent quasi-random sample of the population of interest.

Finally, if the observed association between obesity and SLC6A14 is causal, what is the biological mechanism and what are the implications? If functioning of the catecholamine neurotransmitter system is involved, does this have implications for the pharmacogenetics of the response to certain antiobesity drugs that act on these systems and certain antipsychotic drugs that cause weight gain (7)? We look 
forward to learning the answers to these questions.

\section{Acknowledgments}

The authors are supported in part by NIH grants R01DK56366, P30DK56336, and R01ES09912.

1. Sturm, R. 2003. Increases in clinically severe obesity in the United States, 1986-2000. Arch. Intern. Med. 163:2146-2148.

2. National Institute of Diabetes \& Digestive \& Kidney Diseases. Phenotyping obesity for human genetic studies. http:/www.niddk.nih.gov/fund/ other/phenotyping/index.

3. Chagnon, Y.C., et al. 2003. The human obesity gene map: the 2002 update. Obes. Res. 11:313-367.

4. Redden, D.T., and Allison, D.B. 2003. Non-replication in genetic association studies of obesity and diabetes. J. Nutr. 133:3323-3326.

5. Rosmond, R. 2003. Association studies of genetic polymorphisms in central obesity: a critical review. Int. J. Obes. Relat. Metab. Disord. 27:1141-1151.

6. Hirschhorn, J.N., and Altshuler, D. 2002. Once and again - issues surrounding replication in genetic association studies. J. Clin. Endocrinol.
Metab. 87:4438-4441.

7. Allison, D.B., et al. 2003. Genetic influences on obesity. In Obesity: mechanisms and clinical management. R. Eckel, editor. Elsevier. New York, New York, USA. 31-74.

8. Segal, N., and Allison, D.B. 2002. Twins and virtual twins: bases of relative body weight revisited. Int. J. Obesity. 26:437-441.

9. Öhman, M., et al. 2000. Genome-wide scan of obesity in Finnish sibpairs reveals linkage to chromosome Xq24. J. Clin. Endocrinol. Metab. 85:3183-3190

10. Suviolahti, E., et al. 2003. The SLC6A14 gene shows evidence of association with obesity. J. Clin. Invest. 112:1762-1772. doi:10.1172/JCI200317491.

11. Sargent, P.A., Sharpley, A.L., Williams, C., Goodall, E.M., and Cowen, P.J. 1997. 5-HT2C receptor activation decreases appetite and body weight in obese subjects. Psychopharmacology (Berl.). 133:309-312.

12. Leibowitz, S.F., and Alexander, J.T. 1998. Hypothalamic serotonin in control of eating behavior, meal size, and body weight. Biol. Psychiatry. 44:851-864.

13. Rankinen, T., and Tiwari, H.K. 2003. Genome scans for human nutritional traits. What have we learned? Nutrition. In press.

14. Hager, J., et al. 1998. A genome-wide scan for human obesity genes reveals a major susceptibility locus on chromosome 10. Nat Genet. 20:304-308.
15. Price, R.A., Li, W.D., and Kilker, R. 2002. An X chromosome scan reveals a locus for fat distribution in chromosome region Xp21-22. Diabetes. 51:1989-1991.

16. Stone, S., et al. 2002. A major predisposition locus for severe obesity, at 4p15-p14. Am.J. Hum Genet. 70:1459-1468.

17. Lander, E., and Kruglyak, L. 1995. Genetic dissection of complex traits: guidelines for interpreting and reporting linkage results. Nat. Genet. 11:241-247.

18. Page, G.P., George, V., Go, R.C., Page, P.Z., and Allison, D.B. 2003. "Are we there yet?": Deciding when one has demonstrated specific genetic causation in complex diseases and quantitative traits. Am. J. Hum. Genet. 73:711-719.

19. Cardon, L.R., and Palmer, L.J. 2003. Population stratification and spurious allelic association. Lancet. 361:598-604.

20. Silventoinen, K., Kaprio, J., Lahelma, E., Viken R.J., and Rose, R.J. 2003. Assortative mating by body height and BMI: Finnish twins and their spouses. Am. J. Human Biol. 15:620-627.

21. Allison, D.B., et al. 2002. Bias in estimates of quantitative-trait-locus effect in genome scans: demonstration of the phenomenon and a method-of-moments procedure for reducing bias. Am. J. Hum. Genet. 70:575-585.

22. Boerwinkle, E., Hixson, J.E., and Hanis, C.L. 2000 Peeking under the peaks: following up genomewide linkage analyses. Circulation. 102:1877-1888.

\section{Genes required for B cell development}

\author{
Mary Ellen Conley 1,2 \\ ${ }^{1}$ Department of Immunology, St. Jude Children's Research Hospital, Memphis, \\ Tennessee, USA \\ ${ }^{2}$ Department of Pediatrics, University of Tennessee College of Medicine, Memphis, \\ Tennessee, USA
}

\begin{abstract}
Mutations in a variety of genes can cause congenital agammaglobulinemia and a failure of B cell development. The currently known genes encode components of the pre-B cell receptor or proteins that are activated by cross-linking of the pre-B cell receptor. Defects in these genes result in a block in $B$ cell differentiation at the pro-B to pre-B cell transition. A patient with a translocation involving a previously unknown gene, $L R R C 8$, demonstrated a block at exactly the same point in B cell differentiation (see the related article beginning on page 1707). It will be interesting to determine whether the protein encoded by this gene interacts with the pre-B cell receptor signal transduction pathway or is involved in a new pathway.
\end{abstract}

J. Clin. Invest. 112:1636-1638 (2003). doi:10.1172/JCI200320408.
B cell maturation proceeds through a series of stages that can be defined by the rearrangement status of the $\mathrm{Ig}$ genes, the expression of cell surface markers, and the location of the cells within the bone marrow, the spleen, or the lymph nodes (1-3). Patients with defects in early B cell maturation usually develop recurrent infections, caused by encapsulated bacteria, in the first 2 years of life, and most are recog- nized to have immunodeficiency when they are hospitalized for a dramatic infection at less than 3 years of age (4). Approximately $80 \%$ of patients with the early onset of recurrent infections, hypogammaglobulinemia, and markedly reduced or absent $B$ cells have X-linked agammaglobulinemia (XLA) (5). This disorder is caused by mutations in a hematopoietic-specific cytoplasmic tyrosine kinase, Btk $(6,7)$. Btk is expressed in myeloid cells, in platelets, and at all stages of B cell development except plasma cells (8-10); however, it is important to note that patients with XLA have absent or markedly reduced numbers of $B$ cells but do not have any clinical abnormalities in myeloid cells or in platelet number or function.

The earliest point in B cell differentiation at which Btk is required coincides with expression of the pre-B cell receptor at the pro- $\mathrm{B}$ to pre-B cell transition $(11,12)$. Therefore, it is not surprising that defects in components of the pre-B cell receptor account for an additional $7-10 \%$ of patients with congenital agammaglobulinemia. The majority of these patients have mutations in the constant region of $\mu$ heavy chain Ig (13), but a small number have defects in $\lambda 5$ (14), which is part of the surrogate light chain, or in $\operatorname{Ig} \alpha(15)$, a transmembrane protein that binds to 